\title{
Factorial states, upper multiplicity and norms of elementary operators
}

\author{
Robert J. Archbold, Douglas W. B. Somerset and Richard M. Timoney
}

\begin{abstract}
Let $\pi$ be an irreducible representation of a $C^{*}$-algebra $A$. We show that the weak* approximation of factorial states associated to $\pi$ by type I factorial states of lower degree is closely related to the value of the upper multiplicity $M_{\mathrm{U}}(\pi)$ of $\pi$. As a consequence, we give a representation-theoretic characterization of those $\mathrm{C}^{*}$-algebras $A$ for which the set of pure states $P(A)$ is weak ${ }^{*}$-closed in the set of factorial states $F(A)$. We also study the matricial norms and the positivity for elementary operators $T$ on $A$. We show that if $M_{\mathrm{U}}(\pi)>1$, then $\left\|T^{\pi}\right\|_{k} \leqslant\|T\|_{n}$ for certain $k>n$, and similarly that the $n$-positivity of $T$ implies the $k$-positivity of $T^{\pi}$ (where $T^{\pi}$ is the induced operator on $\pi(A))$. We use these localizations at $\pi$ to give new proofs of various characterizations of the class of antiliminal-by-abelian $\mathrm{C}^{*}$-algebras in terms of factorial states and elementary operators. In the course of this, we show that antiliminal-by-abelian is equivalent to abelian-by-antiliminal.
\end{abstract}

\section{Introduction}

A $\mathrm{C}^{*}$-algebra $A$ is said to be antiliminal-by-abelian $[\mathbf{9}]$ if it is an extension of an antiliminal $\mathrm{C}^{*}$-algebra by an abelian one. That is, either $A$ has an abelian, proper, closed, two-sided ideal $J$ (possibly $\{0\}$ ) such that $A / J$ is antiliminal or else $A$ is abelian. This class of algebras first appeared in $[\mathbf{1 1}]$ as being precisely the class for which the set of pure states $P(A)$ is weak*-dense in the set of factorial states $F(A)$. Subsequently, it was shown in $[\mathbf{9}]$ that the antiliminal-by-abelian $\mathrm{C}^{*}$-algebras also exactly solve two problems concerning elementary operators on $\mathrm{C}^{*}$-algebras. That is, they are precisely the algebras for which every positive elementary operator is completely positive, and they are also the algebras for which the $c b$-norm of every elementary operator coincides with the operator norm. Thus the antiliminal-by-abelian algebras could be seen to form an unexpected link between properties of elementary operators and the approximation of factorial states.

In $[\mathbf{2 1}]$, new techniques made this link direct. In [21, Theorem 1.4], it was shown that the $k$-positivity of an elementary operator can be tested by using factorial states for which the associated Gelfand-Naimark-Segal (GNS) commutant is of type $\mathrm{I}_{j}$ for $j \leqslant k$. From this, it was shown that a certain approximation property of factorial states (which had previously been shown equivalent to a notion of antiliminal by $k$-subhomogeneous in [11, Theorem 4.5]) exactly characterizes those $\mathrm{C}^{*}$-algebras for which every $k$-positive elementary operator is completely positive [21, Corollary 4.6]. A further equivalent condition is that the $c b$-norm of every elementary operator is equal to the $k$-norm [22, Theorem 4.5].

In this paper, we establish a new perspective on the links between factorial states, representation theory and elementary operators by localizing in the spectrum $\hat{A}$ of $A$. We begin by considering the approximation of factorial states associated with a fixed irreducible representation $\pi$ by type-I factorial states of lower degree (where a type-I factorial state $\phi$

Received 21 November 2007; published online 27 August 2008.

2000 Mathematics Subject Classification 46L05, 46L07, 46L30, 47B47.

The third author was supported in part by Science Foundation Ireland under grant 05/RFP/MAT0033. 
is said to have degree $k$ if the GNS commutant $\pi_{\phi}(A)^{\prime}$ is a factor of type $\left.\mathrm{I}_{k}(1 \leqslant k \leqslant \infty)\right)$. We show that such approximation is closely related to the value of the upper multiplicity $M_{\mathrm{U}}(\pi)$ of $\pi$ (Theorem 2.1). From this, we are able to deduce an extensive list of corollaries concerning the approximation of (factorial) states by type-I factorial states of specified degree.

In particular, by restricting the approximating states to be pure (that is, of degree 1 ), we show that $P(A)$ is closed in $F(A)$ if and only if $\pi$ is a Fell point for all $\pi \in \hat{A}$ for which $\operatorname{dim} \pi>1$ (Corollary 2.6(a)). This result combines with an earlier characterization of the condition $\overline{P(A)} \cap$ $S(A) \subseteq F(A)$ (see [3, Corollary; 11, Theorem 3.5]) to recover Glimm's description of those $\mathrm{C}^{*}$ algebras for which $P(A)$ is closed in the state space $S(A)$ [16, Theorem 6]. This dissection of Glimm's theorem is somewhat analogous to the way in which the Glimm-Tomiyama-Takesaki characterization of the condition $\overline{P(A)} \supseteq S(A)[\mathbf{1 5}, \mathbf{2 4}]$ may be viewed as a combination of [2, Theorem 3.3; 11, Theorem 3.4] (see the discussion in [11, pp. 131, 136]). We also show that if the set $F_{n}(A)$, consisting of all those type I factorial states with degree at most $n$, is closed in $F(A)$ (or even in $S(A)$ ) for some positive integer $n$ then so is $F_{k}(A)$ for all integers $k \geqslant n$.

In Section 3, we turn to the estimation of the matricial norms of an elementary operator $T$ on $A$ and of the induced operator $T^{\pi}$ on $\pi(A)$ (see below). In Theorem 3.1, we apply Theorem 2.1 and the notion of tracial geometric mean from [23] to obtain inequalities $\left\|T^{\pi}\right\|_{k} \leqslant\|T\|_{n}$ for certain values of $k$ and $n$ related to $M_{\mathrm{U}}(\pi)$. By using [21, Theorem 1.4], we also show that the $n$-positivity of $T$ implies the $k$-positivity of $T^{\pi}$. The remainder of Section 3 is primarily concerned with obtaining partial converses to Theorem 3.1. This concludes with Theorem 3.8 which shows that the failure of Fell's condition for an irreducible representation $\pi$ is very closely related to the inequalities $\left\|T^{\pi}\right\|_{n+1} \leqslant\|T\|_{n}$ and also to analogous conditions on matricial positivity.

In Section 4, we show that our localized techniques are strong enough to recover the results of $[\mathbf{1 1}, \mathbf{2 1}, \mathbf{2 2}]$ discussed above. In particular, Theorem 2.1 leads to an alternative proof of [11, Theorem 4.5], and Theorem 2.1 and Corollary 3.7 lead to an alternative proof of $[\mathbf{2 2}$, Corollary 4.5] and the corresponding parts of [21, Corollary 4.6]. These proofs are combined in Theorem 4.3. In preparation for this, we establish two elementary results which may be of independent interest. The first (Proposition 4.1) draws on [4] to give simple characterizations of antiliminal $\mathrm{C}^{*}$-algebras in terms of the upper multiplicity of irreducible representations. The second (Proposition 4.2) includes the elementary, but apparently new, observation that 'antiliminal-by-abelian' is equivalent to 'abelian-by-antiliminal'.

We end this section with some notation and preliminaries. We recall that if $A$ is a $\mathrm{C}^{*}$-algebra and $\pi \in \hat{A}$, then $\pi$ is said to be a Fell point if there exist a neighbourhood $V$ of $\pi$ and an element $a \in A$ such that $\sigma(a)$ is a projection of rank 1 for every $\sigma \in V[\mathbf{1 4}$, Section 4.1]. One of the reasons for introducing the upper multiplicity $M_{\mathrm{U}}(\pi)$ of $\pi$ in [4] was to quantify the extent to which Fell's condition may fail to hold. It was shown in [4, Theorem 4.6] that $\pi$ is a Fell point if and only if $M_{\mathrm{U}}(\pi)=1$. A convenient characterization of the inequality $M_{\mathrm{U}}(\pi) \geqslant k$, in terms of the convergence of pure states, is given in [6, Lemma 2.1].

Let $F_{\infty}(A)$ be the set of all states $\phi$ of a $\mathrm{C}^{*}$-algebra $A$ for which $\pi_{\phi}(A)^{\prime}$ is a type-I factor. See [11, Section 2] for a detailed discussion of such states. In particular, there is a continuous, open map $\theta: F_{\infty}(A) \rightarrow \hat{A}$ such that $\theta(\phi)$ is the unique unitary equivalence class in $\hat{A}$ whose members are quasi-equivalent to the GNS-representation $\pi_{\phi}$. It follows from [11, Proposition 2.1] that if $\pi \in \hat{A}$ and if $\operatorname{dim} \pi \leqslant m$, then $F_{\infty}(A) \cap \theta^{-1}(\pi)=F_{m}(A) \cap$ $\theta^{-1}(\pi)$.

An elementary operator $T$ on a $\mathrm{C}^{*}$-algebra $A$ has the form $T(x)=\sum_{i=1}^{n} a_{i} x b_{i}(x \in A)$, where $n \geqslant 1$ and $a_{1}, b_{1}, \ldots, a_{n}, b_{n}$ belong to the multiplier algebra $M(A)$. If $\pi$ is an irreducible representation of $A$ on a Hilbert space $H_{\pi}$, then there is a unique extension to an irreducible representation $\bar{\pi}$ of $M(A)$ on $H_{\pi}$. An elementary operator $T$ on $A$ (as above) induces an 
elementary operator $T^{\pi}$ on $\pi(A)$ given by the formula

$$
T^{\pi}(y)=\sum_{i=1}^{n} \bar{\pi}\left(a_{i}\right) y \bar{\pi}\left(b_{i}\right) \quad(y \in \pi(A)) .
$$

If an elementary operator $T$ on $A$ can be written in the form $T=\sum_{i=1}^{n} a_{i}(\cdot) b_{i}$ for some $n$ and $a_{1}, b_{1}, \ldots, a_{n}, b_{n} \in A$, then we say that $T$ is an elementary operator with coefficients in $A$.

\section{Factorial states and upper multiplicity}

Theorem 2.1, the main result of this section, shows how the upper multiplicity of an irreducible representation $\pi$ is related to the approximation of factorial states associated to $\pi$ by type-I factorial states of lower degree. Condition (1) is apparently stronger than the simpler condition (2) but is, in fact, equivalent to it. The reason for including (1) is that it is needed for an application to the norms of elementary operators (see Theorem 3.1).

Theorem 2.1. Let $A$ be a $\mathrm{C}^{*}$-algebra, let $1 \leqslant n \leqslant m<\infty$ and let $\pi \in \hat{A}$. Consider the following four conditions:

(1) Whenever $\phi_{1}, \phi_{2} \in F_{m+1}(A)$ satisfy $\theta\left(\phi_{1}\right)=\theta\left(\phi_{2}\right)=\pi$, there exist nets $\left(\phi_{\lambda}^{(1)}\right)_{\lambda \in \Lambda}$ and $\left(\phi_{\lambda}^{(2)}\right)_{\lambda \in \Lambda}$ in $F_{n}(A)$ such that $\theta\left(\phi_{\lambda}^{(1)}\right)=\theta\left(\phi_{\lambda}^{(2)}\right)$ for each $\lambda$ and $\phi_{\lambda}^{(i)} \rightarrow_{\lambda} \phi_{i}(i=1,2)$.

(2) $\left\{\phi \in F_{m+1}(A): \theta(\phi)=\pi\right\} \subseteq \overline{F_{n}(A)}$;

(3) $\left\{\phi \in F_{\infty}(A) \backslash F_{m}(A): \theta(\phi)=\pi\right\} \cap \overline{F_{n}(A)}$ is non-empty;

(4) $M_{\mathrm{U}}(\pi)>m / n$.

Then $(3) \Rightarrow(4) \Rightarrow(1) \leftrightarrow(2)$. If $\operatorname{dim} \pi>m$, then all four conditions are equivalent. If condition (3) holds, then $\operatorname{dim} \pi>m$.

Proof. The implication (1) $\Rightarrow(2)$ is immediate. If $\operatorname{dim} \pi>m$, then $\left\{\phi \in F_{m+1}(A) \backslash F_{m}(A)\right.$ : $\theta(\phi)=\pi\}$ is non-empty and so (2) implies (3).

Now suppose that (3) holds but that $M_{\mathrm{U}}(\pi) \leqslant k$, the integer part of $m / n$. By (3), there exists $\phi \in F_{\infty}(A) \backslash F_{m}(A)$ such that $\theta(\phi)=\pi$ and $\phi \in \overline{F_{n}(A)}$. Since $\phi \in F_{\infty}(A) \backslash F_{m}(A)$ and $\theta(\phi)=$ $\pi$, there is a countable set $S$ of positive integers, containing $\{1, \ldots, m+1\}$, and a set $\left\{\xi_{i}\right.$ : $i \in S\}$ of non-zero mutually orthogonal vectors in $H_{\pi}$ such that $\phi=\sum_{i \in S}\left\langle\pi(\cdot) \xi_{i}, \xi_{i}\right\rangle$ (see [11, Proposition 2.1, proof of (i) and statement of (ii)]). On the other hand, since $\phi \in \overline{F_{n}(A)}$, there is a net $\left(\phi_{\lambda}\right)$ in $F_{n}(A)$ such that $\phi=\lim \phi_{\lambda}$. By continuity, $\pi_{\lambda}:=\theta\left(\phi_{\lambda}\right) \rightarrow \pi$ in $\hat{A}$.

Since $M_{\mathrm{U}}(\pi) \leqslant k$, it follows from [8, Theorem 2.5(ii)] that there exist $q \in A^{+}$and a neighbourhood $V$ of $\pi$ in $\hat{A}$ such that $\pi(q)$ is a rank one projection and $\operatorname{rank}(\sigma(q)) \leqslant k$ for all $\sigma \in \hat{A}$. Since $\pi_{\lambda} \rightarrow \pi$, we may assume that $\pi_{\lambda} \in V$ for all $\lambda$. Let $\xi$ be a unit vector in $H_{\pi}$ such that $\pi(q) \xi=\xi$. By Kadison's transitivity theorem, there exists $a_{j} \in A$ such that $\pi\left(a_{j}\right) \xi=\xi_{j}$ for $1 \leqslant j \leqslant n k+1$ (note that $n k+1 \leqslant m+1$ ). Since $\phi_{\lambda} \rightarrow \phi$, we have

$$
\operatorname{det}\left(\left[\phi_{\lambda}\left(a_{j} q a_{i}^{*}\right)\right]_{i, j=1}^{n k+1}\right) \longrightarrow_{\lambda} \operatorname{det}\left(\left[\phi\left(a_{j} q a_{i}^{*}\right)\right]_{i, j=1}^{n k+1}\right) .
$$

Now temporarily fix $\lambda$. Since $\phi_{\lambda} \in F_{n}(A)$ and $\pi_{\lambda}(q)$ has rank at most $k$, the matrix $\left[\phi_{\lambda}\left(a_{j} q a_{i}^{*}\right)\right]_{i, j=1}^{n k+1}$ can be expressed as a linear combination of $n k$ or fewer matrices of the form $\left[\left\langle\pi_{\lambda}\left(a_{j}\right) e \pi_{\lambda}\left(a_{i}^{*}\right) \eta, \eta\right\rangle\right]_{i, j=1}^{n k+1}$, where $\eta \in H_{\pi_{\lambda}}$ and $e$ is a rank one projection. However, the latter matrix has rank at most 1 because if $v$ is a unit vector such that $e v=v$ then $e \pi_{\lambda}\left(a_{i}^{*}\right) \eta=t_{i} v$ for some scalar $t_{i}$ and so $\left\langle\pi_{\lambda}\left(a_{j}\right) e \pi_{\lambda}\left(a_{i}^{*}\right) \eta, \eta\right\rangle=t_{i} \overline{t_{j}}$. Thus the matrix $\left[\phi_{\lambda}\left(a_{j} q a_{i}^{*}\right)\right]_{i, j=1}^{n k+1}$ has rank at most $n k$ and hence has zero determinant.

It follows that

$$
\operatorname{det}\left(\left[\phi\left(a_{j} q a_{i}^{*}\right)\right]_{i, j=1}^{n k+1}\right)=0 .
$$


On the other hand,

$$
\begin{aligned}
\phi\left(a_{j} q a_{i}^{*}\right) & =\sum_{r \in S}\left\langle\pi\left(a_{j}\right) \pi(q) \pi\left(a_{i}^{*}\right) \xi_{r}, \xi_{r}\right\rangle \\
& =\left\langle\pi\left(a_{j}\right) \pi(q) \pi\left(a_{i}^{*}\right) \xi_{j}, \xi_{j}\right\rangle \\
& =\left\langle\xi_{j}, \pi\left(a_{i}\right) \pi(q) \pi\left(a_{j}\right)^{*} \xi_{j}\right\rangle .
\end{aligned}
$$

Thus the matrix $\left[\phi\left(a_{j} q a_{i}^{*}\right)\right]_{i, j=1}^{n k+1}$ is diagonal, with $(i, i)$-entry equal to $\left\|\xi_{i}\right\|^{4}$. Thus the determinant is non-zero, giving the required contradiction.

$(4) \Rightarrow(1)$. Assume that (4) holds and suppose that $\phi_{1}, \phi_{2} \in F_{m+1}(A)$ satisfy $\theta\left(\phi_{1}\right)=\theta\left(\phi_{2}\right)=$ $\pi$. Let $k$ be a positive integer such that $M_{\mathrm{U}}(\pi) \geqslant k>m / n$, and let $\xi$ be a unit vector in $H_{\pi}$. By [6, Lemma 2.1], there exist a net $\left(\pi_{\lambda}\right)$ in $\hat{A}$ and, for each $\lambda$, an orthonormal set $\left\{\eta_{1, \lambda}, \ldots, \eta_{k, \lambda}\right\}$ such that

$$
\left\langle\pi_{\lambda}(\cdot) \eta_{j, \lambda}, \eta_{j, \lambda}\right\rangle \longrightarrow\langle\pi(\cdot) \xi, \xi\rangle \quad(1 \leqslant j \leqslant k) .
$$

Let $\tilde{A}=A$, if $A$ is unital, and $\tilde{A}=A+\mathbb{C} 1$, if $A$ is non-unital. For each $\sigma \in \hat{A}$, let $\tilde{\sigma}$ be the unique extension of $\sigma$ to $\tilde{A}$. Then, if $j \neq l$, it follows from [7, Lemma 2.5] that

$$
\left\langle\pi_{\lambda}(a) \eta_{j, \lambda}, \eta_{l, \lambda}\right\rangle \longrightarrow 0 \quad(a \in \tilde{A}) .
$$

Since $\phi_{1} \in F_{m+1}(A)$ and $\theta\left(\phi_{1}\right)=\pi$, there exist $p \in\{1,2, \ldots, m+1\}$, an orthonormal set $\left\{\xi_{1}, \ldots, \xi_{p}\right\}$ in $H_{\pi}$ and positive scalars $t_{i}, \ldots, t_{p}$ such that $\sum_{i=1}^{p} t_{i}=1$ and $\phi_{1}=$ $\sum_{i \overline{\overline{\tilde{A}}} 1}^{p} t_{i}\left\langle\pi(\cdot) \xi_{i}, \xi_{i}\right\rangle$. By Kadison's transitivity theorem, there exist unitary elements $u_{1}, \ldots, u_{p}$ in $\tilde{\tilde{A}}$ such that $\tilde{\pi}\left(u_{i}\right) \xi=\xi_{i}$ for $1 \leqslant i \leqslant p$.

By a variant of the usual division algorithm, $p=q k-r$, where $q \geqslant 1$ and $0 \leqslant r<k$. Then $q \leqslant n$. If $q \geqslant 2$ and $1 \leqslant j \leqslant q-1$, then we define $v_{j, \lambda}=\sum_{i=1}^{k} t_{i+(j-1) k}^{1 / 2} \tilde{\pi}_{\lambda}\left(u_{i+(j-1) k}\right) \eta_{i, \lambda}$. Also, whatever the value of $q$, we define

$$
v_{q, \lambda}=\sum_{i=1}^{k-r} t_{i+(q-1) k}^{1 / 2} \tilde{\pi}_{\lambda}\left(u_{i+(q-1) k}\right) \eta_{i, \lambda} .
$$

If $1 \leqslant j \leqslant q-1$, then it follows from $(2.2)$ that

$$
\begin{aligned}
\left\|v_{j, \lambda}\right\|^{2}= & \left\langle\sum_{i=1}^{k} t_{i+(j-1) k}^{1 / 2} \tilde{\pi}_{\lambda}\left(u_{i+(j-1) k}\right) \eta_{i, \lambda}, \sum_{l=1}^{k} t_{l+(j-1) k}^{1 / 2} \tilde{\pi}_{\lambda}\left(u_{l+(j-1) k}\right) \eta_{l, \lambda}\right\rangle \\
& \longrightarrow_{\lambda} \lim _{\lambda} \sum_{i=1}^{k} t_{i+(j-1) k}\left\langle\tilde{\pi}_{\lambda}\left(u_{i+(j-1) k}\right) \eta_{i, \lambda}, \tilde{\pi}_{\lambda}\left(u_{i+(j-1) k}\right) \eta_{i, \lambda}\right\rangle \\
= & \sum_{i=1}^{k} t_{i+(j-1) k}=\mu_{j} \quad \text { (say). }
\end{aligned}
$$

Similarly

$$
\left\|v_{q, \lambda}\right\|^{2} \longrightarrow \lambda \sum_{i=1}^{k-r} t_{i+(q-1) k}=\mu_{q} \quad \text { (say). }
$$

Therefore there exists a $\lambda_{1}$ such that, for $\lambda \geqslant \lambda_{1}$ and $1 \leqslant j \leqslant q, v_{j, \lambda} \neq 0$. Therefore, we may define

$$
w_{j, \lambda}=\frac{v_{j, \lambda}}{\left\|v_{j, \lambda}\right\|} \quad\left(\lambda \geqslant \lambda_{1}, 1 \leqslant j \leqslant q\right) .
$$

For $\lambda \geqslant \lambda_{1}$, we define

$$
\phi_{\lambda}^{(1)}=\sum_{j=1}^{q} \mu_{j}\left\langle\pi_{\lambda}(\cdot) w_{j, \lambda}, w_{j, \lambda}\right\rangle \in F_{n}(A)
$$


(recall that $q \leqslant n$ and note that $\sum_{j=1}^{q} \mu_{j}=\sum_{i=1}^{p} t_{i}=1$ ). Let $a \in A$. Using (2.3) and (2.4), and then (2.1) and (2.2), we obtain that

$$
\begin{aligned}
\phi_{\lambda}^{(1)}(a) & \longrightarrow{ }_{\lambda} \lim _{\lambda} \sum_{j=1}^{q}\left\langle\pi_{\lambda}(a) v_{j, \lambda}, v_{j, \lambda}\right\rangle \\
= & \sum_{j=1}^{q-1} \sum_{i=1}^{k} t_{i+(j-1) k}\left\langle\pi(a) \tilde{\pi}\left(u_{i+(j-1) k}\right) \xi, \tilde{\pi}\left(u_{i+(j-1) k}\right) \xi\right\rangle \\
& +\sum_{i=1}^{k-r} t_{i+(q-1) k}\left\langle\pi(a) \tilde{\pi}\left(u_{i+(q-1) k}\right) \xi, \tilde{\pi}\left(u_{i+(q-1) k}\right) \xi\right\rangle \\
= & \sum_{i=1}^{p} t_{i}\left\langle\pi(a) \xi_{i}, \xi_{i}\right\rangle=\phi_{1}(a) .
\end{aligned}
$$

Similarly, we may find $\lambda_{2} \geqslant \lambda_{1}$ and construct $\phi_{\lambda}^{(2)} \in F_{n}(A)$ for all $\lambda \geqslant \lambda_{2}$ such that $\theta\left(\phi_{\lambda}^{(2)}\right)=$ $\pi_{\lambda}=\theta\left(\phi_{\lambda}^{(1)}\right)$ for all $\lambda \geqslant \lambda_{2}$ and $\phi_{\lambda}^{(2)} \rightarrow_{\lambda} \phi_{2}$.

(2) $\Rightarrow(1)$. Assume (2) and let $N=\operatorname{dim} \pi$. We have already seen that if $N>m$ then (1) holds. Also, if $N \leqslant n$ then $\left\{\phi \in F_{m+1}(A): \theta(\phi)=\pi\right\} \subseteq F_{N}(A) \subseteq F_{n}(A)$ and so (1) holds. Therefore we may assume that $n<N \leqslant m$. Then (2) holds with $m$ replaced by $N-1$. Since $\operatorname{dim} \pi>$ $N-1$, (1) holds with $m$ replaced by $N-1$. Since $\left\{\phi \in F_{m+1}(A): \theta(\phi)=\pi\right\} \subseteq F_{N}(A)$, we see that (1) holds as required.

Finally, if (3) holds then, in particular, $\left\{\phi \in F_{\infty}(A) \backslash F_{m}(A): \theta(\phi)=\pi\right\}$ is non-empty and so $\operatorname{dim} \pi>m$.

Corollary 2.2. Let $A$ be a $\mathrm{C}^{*}$-algebra, and let $1 \leqslant n \leqslant m<\infty$. The following conditions are equivalent:

(1) $\overline{F_{n}(A)} \cap F(A) \subseteq F_{m}(A)$;

(2) $\overline{F_{n}(A)} \cap F_{\infty}(A) \subseteq F_{m}(A)$;

(3) $M_{U}(\pi) \leqslant m / n$ for all $\pi \in \hat{A}$ for which $\operatorname{dim} \pi>m$.

Proof. Since $F_{\infty}(A) \subseteq F(A)$, it is clear that (1) implies (2).

Suppose that (2) holds and suppose that $\pi \in \hat{A}$ satisfies $\operatorname{dim} \pi>m$. Then it follows from Theorem $2.1((4) \Rightarrow(3))$ that $M_{\mathrm{U}}(\pi) \leqslant m / n$.

Finally, suppose that (3) holds. Let $U=\{\pi \in \hat{A}: \operatorname{dim} \pi>m\}$, an open subset of $\hat{A}[\mathbf{1 3}$, 3.6.3(i)], and let $J$ be the ideal of $A$ such that $\hat{J}=U$ (so that $J=\{0\}$ if $U$ is empty). Then $A / J$ is $m$-subhomogeneous and $J$ is liminal [4, Corollary 4.9]. Hence $A$ is postliminal and so $F(A)=F_{\infty}(A)$. Let $\phi \in \overline{F_{n}(A)} \cap F(A)$ and let $\pi=\theta(\phi)$. If $\operatorname{dim} \pi \leqslant m$, then $\phi \in F_{m}(A)$. On the other hand, if $\operatorname{dim} \pi>m$, then $M_{\mathrm{U}}(\pi) \leqslant m / n$ and so $\phi \in F_{m}(A)$ by Theorem 2.1 $((3) \Rightarrow(4))$.

The next result is a special case of Corollary 2.2 obtained by taking $n=1$.

Corollary 2.3. Let $A$ be a $\mathrm{C}^{*}$-algebra, and let $m$ be a positive integer. The following conditions are equivalent:

(1) $\overline{P(A)} \cap F(A) \subseteq F_{m}(A)$;

(2) $\overline{P(A)} \cap F_{\infty}(A) \subseteq F_{m}(A)$;

(3) $M_{\mathrm{U}}(\pi) \leqslant m$ for all $\pi \in \hat{A}$ for which $\operatorname{dim} \pi>m$. 
Corollary 2.4. Let $A$ be a $\mathrm{C}^{*}$-algebra, and let $m$ be a positive integer. The following conditions are equivalent:

(1) $\overline{F_{m}(A)} \cap F(A)=F_{m}(A)$;

(2) $\overline{F_{m}(A)} \cap F_{\infty}(A)=F_{m}(A)$;

(3) $\pi$ is a Fell point for all $\pi \in \hat{A}$ for which $\operatorname{dim} \pi>m$;

(4) $\overline{F_{[m / 2]+1}(A)} \cap F(A) \subseteq F_{m}(A)$;

(5) $\overline{F_{[m / 2]+1}(A)} \cap F_{\infty}(A) \subseteq F_{m}(A)$.

Furthermore, if $F_{m}(A)$ is closed in $F(A)$, then $F_{k}(A)$ is closed in $F(A)$ for all $k \geqslant m$.

Proof. We begin by recalling from [4, Theorem 4.6] that, for $\pi \in \hat{A}, M_{\mathrm{U}}(\pi)=1$ if and only if $\pi$ is a Fell point. Then the equivalence of (1), (2) and (3) follows from Corollary 2.2 by taking $n=m$, and the equivalence of (3), (4) and (5) follows by taking $n=[m / 2]+1$ (in which case $M_{\mathrm{U}}(\pi) \leqslant m / n$ if and only if $M_{\mathrm{U}}(\pi)=1$ ). The final part of the statement follows from the fact that if (3) holds then it also holds when $m$ is replaced by any $k \geqslant m$.

The equivalence of (1) and (3) in the next result has been previously obtained by Larry Brown (private communication). Recall that $S(A)$ is the state space of a $\mathrm{C}^{*}$-algebra $A$.

Corollary 2.5. Let $A$ be a $\mathrm{C}^{*}$-algebra, and let $m$ be a positive integer. The following conditions are equivalent:

(1) $\overline{F_{m}(A)} \cap S(A)=F_{m}(A)$;

(2) $\overline{F_{[m / 2]+1}(A)} \cap S(A) \subseteq F_{m}(A)$;

(3) $A$ is liminal, $\hat{A}$ is Hausdorff, and $\pi$ is a Fell point for all $\pi \in \hat{A}$ for which $\operatorname{dim} \pi>m$;

(4) $\hat{A}$ is Hausdorff, and $\pi$ is a Fell point for all $\pi \in \hat{A}$ for which $\operatorname{dim} \pi>m$.

Furthermore, if $F_{m}(A)$ is closed in $S(A)$, then $F_{k}(A)$ is closed in $S(A)$ for all $k \geqslant m$.

Proof. It is clear that (1) implies (2). Suppose next that (2) holds. Then, in particular, $\overline{P(A)} \cap S(A) \subseteq F(A)$. Hence $A$ is liminal and $\hat{A}$ is Hausdorff by [11, Theorem 3.5] together with $[\mathbf{3}$, p. 252]. The remainder of item (3) follows from Corollary $2.4((4) \Rightarrow(3))$.

Now suppose that (3) holds. Since $A$ is liminal and $\hat{A}$ is Hausdorff, it follows from $[\mathbf{2 0}$, Proposition 9] (see also [2, Theorem 5.2]) that $\overline{F(A)} \cap S(A)=F(A)$. Hence (1) holds by Corollary $2.4((3) \Rightarrow(1))$.

Clearly (3) implies (4), so assume now that (4) holds. Since the set of $\pi \in \hat{A}$ for which $\operatorname{dim} \pi>m$ is open $[\mathbf{1 3}, 3.6 .3(\mathrm{i})], A$ has a closed two-sided Fell ideal $J$ such that $A / J$ is $m$-subhomogeneous. Hence $A$ is a type-I $\mathrm{C}^{*}$-algebra. Since $\hat{A}$ is Hausdorff, $A$ is liminal.

The final part of the statement follows from the fact that if (3) holds then it also holds when $m$ is replaced by any $k \geqslant m$.

By taking $m=1$ in Corollaries 2.3 and 2.5, we obtain the following results for pure states. Part (b) is (a slight variant of) a well-known theorem of Glimm [16, Theorem 6] (see the discussion in $[\mathbf{1 1}$, p. 136]).

Corollary 2.6. Let $A$ be a $C^{*}$-algebra.

(a) The following conditions are equivalent:

(1) $\overline{P(A)} \cap F(A)=P(A)$;

(2) $\overline{P(A)} \cap F_{\infty}(A)=P(A)$;

(3) $\pi$ is a Fell point for all $\pi \in \hat{A}$ for which $\operatorname{dim} \pi>1$.

(b) The following conditions are equivalent: 
(1) $\overline{P(A)} \cap S(A)=P(A)$;

(2) $A$ is liminal, $\hat{A}$ is Hausdorff, and $\pi$ is a Fell point for all $\pi \in \hat{A}$ for which $\operatorname{dim} \pi>1$.

Glimm's theorem is used in the proof of [11, Theorem 4.5]. We shall show in Section 4 that the latter result can alternatively be obtained by using Theorem 2.1 .

\section{Upper multiplicity and elementary operators}

Theorem 3.1. Let $A$ be a $C^{*}$-algebra, let $\pi \in \hat{A}$ and let $n$ be a positive integer. Let $k$ be any positive integer such that $k \leqslant n M_{\mathrm{U}}(\pi)$ (where it is understood that $n \times \infty=\infty$ ).

(1) For every elementary operator $T$ on $A,\left\|T^{\pi}\right\|_{k} \leqslant\|T\|_{n}$.

(2) For every $n$-positive elementary operator $T$ on $A, T^{\pi}$ is $k$-positive.

Proof. If $M_{\mathrm{U}}(\pi)=1$, then (1) and (2) are clear, so we assume that $M_{\mathrm{U}}(\pi)>1$. Then it is sufficient to prove (1) and (2) for $k=r n$, a multiple of $n$ with $1<r \leqslant M_{\mathrm{U}}(\pi)$. We will do this by applying Theorem $2.1\left((4) \Rightarrow(1)\right.$ ) with $m=k-1$ (so that $M_{\mathrm{U}}(\pi) \geqslant k / n>m / n$ ).

We begin by recalling that the set of pure states associated to $\pi$ is weak*-dense in $P(\pi(A))$ (see, for example, $[\mathbf{1 3}, 3.4 .2(\mathrm{ii})]$ ). It follows that $\left\{\phi \in F_{m+1}(A): \theta(\phi)=\pi\right\}$ is weak*-dense in $F_{m+1}(\pi(A))$. Thus, given $\phi_{1}, \phi_{2} \in F_{m+1}(\pi(A))$, it follows from (1) of Theorem 2.1 that there exist commonly-indexed nets $\left(\phi_{\lambda}^{(1)}\right)_{\lambda \in \Lambda}$ and $\left(\phi_{\lambda}^{(2)}\right)_{\lambda \in \Lambda}$ in $F_{n}(A)$ such that $\theta\left(\phi_{\lambda}^{(1)}\right)=\theta\left(\phi_{\lambda}^{(2)}\right)$ for each $\lambda$ and $\phi_{\lambda}^{(i)} \rightarrow_{\lambda} \phi_{i} \circ \pi(i=1,2)$.

Let $\bar{\pi}$ be the unique extension of $\pi$ to an irreducible representation of $M(A), \bar{\phi}_{\lambda}^{(i)}$ the unique extension of $\phi_{\lambda}^{(i)}$ to a state of $\left.M(A)\right)$ and $\bar{\phi}_{i}$ the unique extension of $\phi_{i}$ to a state of $\bar{\pi}(M(A))$ $(i=1,2)$. Since the map from $S(A)$ into $S(M(A))$ defined by unique extension of states is weak*-continuous, $\bar{\phi}_{\lambda}^{(i)} \rightarrow_{\lambda} \bar{\phi}_{i} \circ \bar{\pi}(i=1,2)$.

Let $T$ be an elementary operator on $A$. Thus $T(x)=\sum_{i=1}^{N} a_{i} x b_{i}(x \in A)$, where $a_{i}, b_{i} \in M(A)$ for $1 \leqslant i \leqslant N$

(1) Using the joint weak*-continuity of tgm as a function of the states (see the proof of $[\mathbf{1 0}$, Lemma 2.1]), we obtain

$$
\operatorname{tgm}\left(\left(Q\left(\left(\bar{\pi}\left(a_{i}^{*}\right)\right)_{i}, \bar{\phi}_{1}\right)\right),\left(Q\left(\left(\bar{\pi}\left(b_{i}\right)\right)_{i}, \bar{\phi}_{2}\right)\right)\right)=\lim _{\lambda} \operatorname{tgm}\left(\left(Q\left(\mathbf{a}^{*}\right), \bar{\phi}_{\lambda}^{(1)}\right),\left(Q(\mathbf{b}), \bar{\phi}_{\lambda}^{(2)}\right)\right) \leqslant\|T\|_{n},
$$

by [23, Corollary 2.2]. Taking the supremum of the left-hand side over pairs $\left(\phi_{1}, \phi_{2}\right)$ satisfying $\theta\left(\phi_{1}\right)=\theta\left(\phi_{2}\right)$, we obtain from $\left[\mathbf{2 3}\right.$, Corollary 2.2] that $\left\|T^{\pi}\right\|_{m+1} \leqslant\|T\|_{n}$.

(2) Suppose that $T$ is $n$-positive and $a \in A$. Then by [21, Theorem 1.4],

$$
\begin{aligned}
\sum_{i=1}^{N} \phi_{1}\left(\bar{\pi}\left(a_{i}\right) \pi(a)^{*}\right) \phi_{1}\left(\pi(a) \bar{\pi}\left(b_{i}\right)\right) & =\sum_{i=1}^{N}\left(\phi_{1} \circ \pi\right)\left(a_{i} a^{*}\right)\left(\phi_{1} \circ \pi\right)\left(a b_{i}\right) \\
& =\lim _{\lambda} \sum_{i=1}^{N} \phi_{\lambda}^{(1)}\left(a_{i} a^{*}\right) \phi_{\lambda}^{(1)}\left(a b_{i}\right) \geqslant 0 .
\end{aligned}
$$

Since this holds for all $a \in A$ and all $\phi_{1} \in F_{k}(\pi(A))$, it follows from [21, Theorem 1.4] that $T^{\pi}$ is $k$-positive.

Corollary 3.2. Let $A$ be a $\mathrm{C}^{*}$-algebra and suppose that $\pi \in \hat{A}$ is a non-Fell point. Let $n \geqslant 1$.

(1) For every elementary operator $T$ on $A,\left\|T^{\pi}\right\|_{n+1} \leqslant\|T\|_{n}$.

(2) For every n-positive elementary operator $T$ on $A, T^{\pi}$ is $(n+1)$-positive. 
Proof. Since $M_{\mathrm{U}}(\pi)>1, n+1 \leqslant 2 n \leqslant n M_{\mathrm{U}}(\pi)$.

For the rest of this section, we consider the possibility of (partial) converses for Theorem 3.1. For example, if $M_{\mathrm{U}}(\pi)=M<\infty$ and $n \geqslant 1$, can we necessarily find an elementary operator $T$ on $A$ such that $\left\|T^{\pi}\right\|_{k}>\|T\|_{n}$ for $k=n M+1$ ? Or, if $M_{\mathrm{U}}(\pi)<\infty$ can we find $n \geqslant 1$ and $T$ with the same property? To see that this does not hold in general, consider the case when $\pi$ is one-dimensional. Then $T^{\pi}$ is always just a scalar and $\left\|T^{\pi}\right\|_{k}=\left\|T^{\pi}\right\| \leqslant\|T\|$ for all $T$ and all $k>1$. More generally, if $\operatorname{dim} \pi \leqslant n M$, then $\left\|T^{\pi}\right\|_{c b}=\left\|T^{\pi}\right\|_{n M}$ and so $\left\|T^{\pi}\right\|_{c b} \leqslant\|T\|_{n}$ by Theorem 3.1. Thus we cannot obtain any converse if $\operatorname{dim} \pi \leqslant n M$, even if we take $n=1$. Therefore we shall suppose that $\operatorname{dim} \pi>n M_{\mathrm{U}}(\pi)$ (which is, of course, automatically satisfied if $\operatorname{dim} \pi=\infty$ ).

We shall show in Theorem 3.6(1) that if we impose the further condition that $\{\pi\}$ is open in

$$
S(\pi):=\{\sigma \in \hat{A}: \pi \text { and } \sigma \text { cannot be separated by disjoint open subsets of } \hat{A}\},
$$

a condition that holds automatically if $M_{\mathrm{U}}(\pi)=1$, then we can find the desired elementary operator $T$ on $A$. This leads easily to Corollary 3.7(1) which is a partial converse to Corollary 3.2(1). Hand-in-hand with these results for norms, we obtain analogous results for positivity.

Before proving Theorem 3.6, we need to establish two lemmas (Lemmas 3.3 and 3.5 below) dealing with elementary operators which behave like certain known examples on a matrix algebra. On the one hand, we have operators that behave like the transpose and, on the other hand, operators that behave like an example due to Choi $[\mathbf{1 2}]$ of an operator $\mathcal{C}_{n}$ on $M_{n}(\mathbb{C})$ which is $(n-1)$-positive but not $n$-positive.

In the proofs, we will use the notation $\mathcal{T}_{n}$ for the transpose mapping on $M_{n}(\mathbb{C})$ and we will use the fact that $\left\|\mathcal{T}_{n}\right\|_{k}=\min \{k, n\}$. Indeed, since $\left\|\mathcal{T}_{n}\right\|=1$, the fact that $\left\|\mathcal{T}_{n}\right\|_{k} \leqslant k$ follows from a general estimate [18, Exercise 3.10 (ii)], while $\left\|\mathcal{T}_{n}\right\|_{k} \geqslant k$ (for $k \leqslant n$ ) follows from a well-known argument $\left[\mathbf{1 8}\right.$, Exercise 1.8]. The fact that $\left\|\mathcal{T}_{n}\right\|_{c b}=\left\|\mathcal{T}_{n}\right\|_{n}$ follows from $[\mathbf{1 8}, 8.11]$. Combining these observations, one obtains the required formula $\left\|\mathcal{T}_{n}\right\|_{k}=\min \{k, n\}$.

The operator $\mathcal{C}_{n}: M_{n} \rightarrow M_{n}$, from [12], is $\mathcal{C}_{n}(x)=(n-1) \operatorname{trace}(x) I_{n}-x$. We note that we can express $\mathcal{T}_{n}$ and $\mathcal{C}_{n}$ as elementary operators by the formulae

$$
\mathcal{T}_{n}(x)=\sum_{i, j=1}^{n} e_{i, j} x e_{i, j}, \mathcal{C}_{n}(x)=\sum_{i, j=1}^{n}(n-1) e_{i, j} x e_{j, i}-\sum_{i, j=1}^{n} e_{i, i} x e_{j, j},
$$

with $\left\{e_{i, j}: 1 \leqslant i, j \leqslant m+1\right\}$ the standard system of matrix units in $M_{m+1}$.

Lemma 3.3. Let $k$ and $m$ be positive integers, and let $H$ be a Hilbert space of dimension $k(m+1)$. Suppose that $\left\{F_{i, j}\right\}_{i, j=1}^{m+1}$ is an $(m+1) \times(m+1)$ system of matrix units in $B(H)$ such that $\sum_{i=1}^{m+1} F_{i, i}=1$. Let $\mathcal{T}$ and $\mathcal{C}$ be the elementary operators on $B(H)$ defined by

$$
\mathcal{T}(S)=\sum_{i, j=1}^{m+1} F_{i, j} S F_{i, j}
$$

and

$$
\mathcal{C}(S)=\sum_{i, j=1}^{m+1} m F_{i, j} S F_{j, i}-\sum_{i, j=1}^{m+1} F_{i, i} S F_{j, j}
$$

(for $S \in B(H)$ ). Then

(i) $\|\mathcal{T}\|=\min \{k, m+1\}$;

(ii) for $r \geqslant 1, \mathcal{C}$ is $r$-positive if and only if $r k \leqslant m$. 
Proof. Let $L$ be the linear span of $\left\{F_{i, j}: 1 \leqslant i, j \leqslant m+1\right\}$. Then we have a ${ }^{*}$-isomorphism $\phi_{1}$ of $M_{m+1}$ onto $L$ given by

$$
\phi_{1}\left(\sum_{i . j=1}^{m+1} \lambda_{i, j} e_{i, j}\right)=\sum_{i . j=1}^{m+1} \lambda_{i, j} F_{i, j} .
$$

Since $L$ is a factor, so is its commutant $L^{\prime}$, and there is a ${ }^{*}$-isomorphism $\Theta$ of $L \otimes L^{\prime}$ onto $B(H)$ given by

$$
\Theta(S \otimes T)=S T \quad\left(S \in L, T \in L^{\prime}\right) .
$$

By considering dimension, the finite-dimensional factor $L^{\prime}$ is ${ }^{*}$-isomorphic to $M_{k}$. Let $\phi_{2}$ be a ${ }^{*}$-isomorphism of $M_{k}$ onto $L^{\prime}$. Thus we have the following sequence of maps:

$$
M_{m+1} \otimes M_{k} \stackrel{\phi_{1} \otimes \phi_{2}}{\longrightarrow} L \otimes L^{\prime} \stackrel{\Theta}{\longrightarrow} B(H) .
$$

Temporarily fix $i, j, r, s \in\{1,2, \ldots, m+1\}$ and define elementary operators $R$ on $B(H)$ and $R_{m+1}$ on $M_{m+1}$ by $R(\cdot)=F_{i, j}(\cdot) F_{r, s}$ and $R_{m+1}(\cdot)=e_{i, j}(\cdot) e_{r, s}$. We claim that

$$
R \circ \Theta \circ\left(\phi_{1} \otimes \phi_{2}\right)=\Theta \circ\left(\phi_{1} \otimes \phi_{2}\right) \circ\left(R_{m+1} \otimes \mathrm{id}_{k}\right),
$$

where $\mathrm{id}_{k}$ is the identity map on $M_{k}$. By linearity, it suffices to check equality on $e_{p, q} \otimes v$, where $v \in M_{k}$. Applying the left-hand side of (3.1), and using the fact that $\phi_{2}(v) \in L^{\prime}$, we obtain

$$
\begin{aligned}
F_{i, j} F_{p, q} \phi_{2}(v) F_{r, s} & =F_{i, j} F_{p, q} F_{r, s} \phi_{2}(v) \\
& =\left(\Theta \circ\left(\phi_{1} \otimes \phi_{2}\right)\right)\left(e_{i, j} e_{p, q} e_{r, s} \otimes v\right),
\end{aligned}
$$

as required. It follows from (3.1) and linearity that

$$
\mathcal{T} \circ \Theta \circ\left(\phi_{1} \otimes \phi_{2}\right)=\Theta \circ\left(\phi_{1} \otimes \phi_{2}\right) \circ\left(\mathcal{T}_{m+1} \otimes \operatorname{id}_{k}\right) .
$$

and

$$
\mathcal{C} \circ \Theta \circ\left(\phi_{1} \otimes \phi_{2}\right)=\Theta \circ\left(\phi_{1} \otimes \phi_{2}\right) \circ\left(\mathcal{C}_{m+1} \otimes \mathrm{id}_{k}\right) .
$$

From (3.2) we have

$$
\|\mathcal{T}\|=\left\|\mathcal{T}_{m+1} \otimes \operatorname{id}_{k}\right\|=\left\|\mathcal{T}_{m+1}\right\|_{k}=\min \{k, m+1\} .
$$

For $r \geqslant 1$, we may tensor both sides of (3.3) with $\mathrm{id}_{r}$ and conclude that

$$
\mathcal{C} \text { is } r \text {-positive } \Longleftrightarrow \mathcal{C}_{m+1} \text { is }(k r) \text {-positive } \Longleftrightarrow k r \leqslant m \text {. }
$$

We denote by $\tau_{s}$ the compact Hausdorff topology defined by Fell [14, Chapter II] on the set $\operatorname{Id}(A)$ of all closed two-sided ideals of a $\mathrm{C}^{*}$-algebra $A$. Note that a net $\left(I_{\alpha}\right)$ is $\tau_{s}$-convergent to $I$ in $\operatorname{Id}(A)$ if and only if $\left\|a+I_{\alpha}\right\| \rightarrow\|a+I\|$ for all $a \in A$ (see [14, Theorem 2.2]). In the proof of the next result, we shall apply [17, Theorem 3.1]. This is a convenient generalization of some classical results on extending matrix units (see [14, Theorem 3.1; 16 Lemmas 10 and $11 ; 24$ p. 506]). We shall also use the notion of a primal ideal from [5].

Lemma 3.4. Let $A$ be a $C^{*}$-algebra and let $1 \leqslant m<\infty$. Suppose that $\pi \in \hat{A}$, that $\operatorname{dim} \pi>m$ and that $M_{\mathrm{U}}(\pi)<\infty$. Let $J$ be a primal ideal of $A$ contained in ker $\pi$. There exist elements $a_{i, j} \in A(1 \leqslant i, j \leqslant m+1)$, and a $\tau_{s}$-neighbourhood $V$ of $J \operatorname{in} \operatorname{Id}(A)$ such that

(i) for each $K \in V,\left\{a_{i, j}+K: 1 \leqslant i, j \leqslant m+1\right\}$ is an $(m+1) \times(m+1)$ system of matrix units in $A / K$

(ii) $\pi\left(a_{1,1}\right)$ has rank 1 ;

(iii) for each $\sigma \in \bigcup_{K \in V} \widehat{A / K}, \sigma\left(a_{1,1}\right)$ has rank at most $M_{\mathrm{U}}(\pi)$ (in $B\left(H_{\sigma}\right)$ ). 
Proof. Since $M_{\mathrm{U}}(\pi)<\infty$, it follows from [4, Theorem 4.4(i)] that $\pi(A)$ contains $\mathcal{K}\left(H_{\pi}\right)$, the algebra of compact linear operators on $H_{\pi}$. Let $\left\{\xi_{1}, \ldots, \xi_{m+1}\right\}$ be an orthonormal set in $H_{\pi}$ and let $E_{i, i}$ be the rank one projection onto the linear span of $\xi_{i}(1 \leqslant i \leqslant m+1)$. For $i \neq j$ let $E_{i, j}$ be the rank one partial isometry which maps $\xi_{j}$ to $\xi_{i}$. Then $\left\{E_{i, j}: 1 \leqslant i, j \leqslant m+1\right\}$ is an $(m+1) \times(m+1)$ system of matrix units in $\pi(A)$. Let $E=\sum_{i=1}^{m+1} E_{i, i}$ and note that there is a *-isomorphism of $M_{m+1}(\mathbb{C})$ onto $E \pi(A) E$ mapping the standard matrix unit $e_{i, j}$ to $E_{i, j}$.

Let $K=\pi^{-1}\left(\mathcal{K}\left(H_{\pi}\right)\right)$, a closed two sided ideal of $A$ which properly contains $J$. Since $M_{\mathrm{U}}(\pi)<\infty$ and $J$ is a primal ideal contained in ker $\pi,\{\pi\}$ is relatively open in $\widehat{A / J}[\mathbf{8}$, Proposition 2.1(ii)] and hence in $\widehat{K / J}$. On the other hand, $\{\pi\}$ is relatively closed in $\widehat{K / J}$ because $\pi(K)$ is simple. Thus there is a ${ }^{*}$-isomorphism $\Psi$ of $\mathcal{K}\left(H_{\pi}\right)$ into $K / J$ such that $\pi \circ \Psi=\operatorname{id}_{\mathcal{K}\left(H_{\pi}\right)}$ and $\sigma \circ \Psi=0$ for all $\sigma \in \widehat{K / J} \backslash\{\pi\}$. Note that if $\sigma \in \widehat{A / J}$ and $\sigma \notin \widehat{K / J}$ then $\sigma \circ \Psi=0$ too.

Let $B=\Psi(E \pi(A) E)$, a finite-dimensional $\mathrm{C}^{*}$-subalgebra of $A / J$. By [17, Theorem 3.1], there is a linear map $L: B \rightarrow A$ and a $\tau_{s}$-neighbourhood $U$ of $J$ in $\operatorname{Id}(A)$ such that

(a) $q_{J} \circ L=\operatorname{id}_{B}$,

(b) for every $K \in U, q_{K} \circ L$ is a ${ }^{*}$-isomorphism of $B$ into $A / K$.

Let $a_{i, j}=L\left(\Psi\left(E_{i, j}\right)\right) \in A(1 \leqslant i, j \leqslant m+1)$. Observe that, by (b), (i) holds for all $\tau_{s}$ neighbourhoods $V \subseteq U$. Regarding $\pi$ in the usual way as an irreducible representation of both $A$ and $A / J$, we have

$$
\pi\left(a_{1,1}\right)=\left(\pi \circ q_{J} \circ L \circ \Psi\right)\left(E_{1,1}\right)=\left(\pi \circ \operatorname{id}_{B} \circ \Psi\right)\left(E_{1,1}\right)=E_{1,1}
$$

and so (ii) holds.

Suppose that there is no $\tau_{s}$-neighbourhood $V$ of $J$ such that (iii) holds. Then there is a net $\left(K_{\alpha}\right)$ in $U$ which is $\tau_{s}$-convergent to $J$ and $\pi_{\alpha} \in \widehat{A / K_{\alpha}}$ such that, for each $\alpha, \operatorname{rank}\left(\pi_{\alpha}\left(a_{1,1}\right)\right)>$ $M_{\mathrm{U}}(\pi)$. Using the $\tau_{s}$-compactness of $\operatorname{Id}(A)$ and passing to a subnet if necessary, we may assume that there exists $I \in \operatorname{Id}(A)$ such that $\operatorname{ker} \pi_{\alpha} \rightarrow I\left(\tau_{s}\right)$. For each $a \in A$,

$$
\|a+J\|=\lim \left\|a+K_{\alpha}\right\| \geqslant \lim \left\|a+\operatorname{ker} \pi_{\alpha}\right\|=\|a+I\| .
$$

Thus $J \subseteq I$.

Let $\xi_{\alpha}$ be a unit vector fixed by the projection $\pi_{\alpha}\left(a_{1,1}\right)$. We shall show that

$$
\left\langle\pi_{\alpha}(\cdot) \xi_{\alpha}, \xi_{\alpha}\right\rangle \rightarrow \phi:=\left\langle\pi(\cdot) \xi_{1}, \xi_{1}\right\rangle .
$$

Let $x \in A$. Then $E_{1,1} \pi(x) E_{1,1}=\lambda E_{1,1}$, where $\lambda=\phi(x)$. Since $q_{J}\left(a_{1,1}\right)=\Psi\left(E_{1,1}\right), \pi\left(a_{1,1}\right.$ $\left.x a_{1,1}-\lambda a_{1,1}\right)=0$. Recalling that $\sigma \circ \Psi=0$ for all $\sigma \in \widehat{A / J} \backslash\{\pi\}$, we obtain $q_{J}\left(a_{1,1} x a_{1,1}-\right.$ $\left.\lambda a_{1,1}\right)=0$. Since $J \subseteq I, q_{I}\left(a_{1,1} x a_{1,1}-\lambda a_{1,1}\right)=0$ and so $\left\|\pi_{\alpha}\left(a_{1,1} x a_{1,1}-\lambda a_{1,1}\right)\right\| \rightarrow 0$. Hence

$$
\lim \left\langle\pi_{\alpha}(x) \xi_{\alpha}, \xi_{\alpha}\right\rangle=\lim \left\langle\pi_{\alpha}\left(a_{1,1} x a_{1,1}\right) \xi_{\alpha}, \xi_{\alpha}\right\rangle=\lim \left\langle\pi_{\alpha}\left(\lambda a_{1,1}\right) \xi_{\alpha}, \xi_{\alpha}\right\rangle=\lambda=\phi(x) .
$$

This establishes (3.4).

Let $\ell=M_{\mathrm{U}}(\pi)+1$. Since $\operatorname{rank}\left(\pi_{\alpha}\left(a_{1,1}\right)\right) \geqslant \ell$, there is an orthonormal set $\left\{\xi_{\alpha}^{(1)}, \ldots, \xi_{\alpha}^{(\ell)}\right\}$ in the range of $\pi_{\alpha}\left(a_{1,1}\right)$ for each $\alpha$. Since $\xi_{\alpha}$ was an arbitrary unit vector fixed by $\pi\left(a_{1,1}\right)$, it follows from (3.4) that for $1 \leqslant i \leqslant \ell,\left\langle\pi_{\alpha}(\cdot) \xi_{\alpha}^{(i)}, \xi_{\alpha}^{(i)}\right\rangle \rightarrow \phi$. Applying [8, Lemma 5.2(i)] to the net $\left(\pi_{\alpha}\right)$, we obtain that

$$
M_{\mathrm{U}}(\pi) \geqslant M_{\mathrm{U}}\left(\pi,\left(\pi_{\alpha}\right)\right) \geqslant \ell=M_{\mathrm{U}}(\pi)+1,
$$

a contradiction. Hence there is a $\tau_{s}$-neighbourhood $V$ of $J$ such that $V \subseteq U$ and (iii) holds.

If $K$ is a closed two-sided ideal of a $\mathrm{C}^{*}$-algebra $A$, then the quotient map $A \rightarrow A / K$ extends uniquely to a *-homomorphism of $M(A)$ into $M(A / K)$. Hence each elementary operator $S$ on $A$ induces an elementary operator $S_{A / K}$ on $A / K$ such that $S_{A / K}(a+K)=S(a)+K(a \in A)$. 
Lemma 3.5. Let $A$ be a $\mathrm{C}^{*}$-algebra and let $1 \leqslant m<\infty$. Suppose that $\pi \in \hat{A}$ and $\operatorname{dim} \pi>$ $m \geqslant M_{\mathrm{U}}(\pi)$. Let $J$ be a primal ideal of $A$ contained in $\operatorname{ker} \pi$.

There exists a $\tau_{s}$-neighbourhood $V$ of $J$ in $\operatorname{Id}(A)$ and elementary operators $T$ and $C$ on $A$ with coefficients in $A$ such that

(i) $\left\|T^{\pi}\right\|_{m+1} \geqslant m+1$ and $C^{\pi}$ is not $(m+1)$-positive;

(ii) for each $K \in V,\left\|T_{A / K}\right\| \leqslant M_{\mathrm{U}}(\pi)$ and $C_{A / K}$ is $r$-positive for $1 \leqslant r \leqslant m / M_{\mathrm{U}}(\pi)$;

(iii) there is a projection $E \in \pi(A)$ of rank $m+1$ with $T^{\pi}(\cdot)=T^{\pi}(E(\cdot) E)$ and $C^{\pi}(\cdot)=$ $C^{\pi}(E(\cdot) E)$.

Proof. There is a $\tau_{s}$-neighbourhood $V$ of $J$ in $\operatorname{Id}(A)$ and elements $a_{i, j} \in A(1 \leqslant i, j \leqslant m+1)$ satisfying (i), (ii) and (iii) of Lemma 3.4. We define

$$
T(x)=\sum_{i, j=1}^{m+1} a_{i, j} x a_{i, j} \quad \text { and } \quad C(x)=\sum_{i, j=1}^{m+1} m a_{i, j} x a_{j, i}-\sum_{i, j=1}^{m+1} a_{i, i} x a_{j, j} \quad(x \in A) .
$$

Let $E_{i, j}=\pi\left(a_{i, j}\right)(1 \leqslant i, j \leqslant m+1)$ and $E=\sum_{i=1}^{m+1} E_{i, i}$, a projection of rank $m+1$. Then

$$
T^{\pi}(\cdot)=\sum_{i, j=1}^{m+1} E_{i, j}(\cdot) E_{i, j} \quad \text { and } \quad C^{\pi}(\cdot)=\sum_{i, j=1}^{m+1} m E_{i, j}(\cdot) E_{j, i}-\sum_{i, j=1}^{m+1} E_{i, i}(\cdot) E_{j, j}
$$

Since $E E_{i, j}=E_{i, j}=E_{i, j} E$ for all $i$ and $j$, condition (iii) is satisfied.

There is an isometric $*$-isomorphism $\Psi$ of $M_{m+1}(\mathbb{C})$ onto $E \pi(A) E$ mapping the standard matrix unit $e_{i, j}$ to $E_{i, j}$. Then $T^{\pi} \circ \Psi=\Psi \circ \mathcal{T}_{m+1}$ and $C^{\pi} \circ \Psi=\Psi \circ \mathcal{C}_{m+1}$. Thus

$$
\left\|T^{\pi}\right\|_{m+1} \geqslant\left\|\left.T^{\pi}\right|_{E \pi(A) E}\right\|_{m+1}=\left\|\mathcal{T}_{m+1}\right\|_{m+1}=m+1
$$

(in fact, equality holds). Also since $\mathcal{C}_{m+1}$ is not $(m+1)$-positive, neither is $\left.C^{\pi}\right|_{E \pi(A) E}$ nor $C^{\pi}$.

To show (ii), let $K \in V$ and $\sigma \in \widehat{A / K}$. Let $k=\operatorname{rank}\left(\sigma\left(a_{1,1}\right)\right) \leqslant M_{\mathrm{U}}(\pi)$ and $F_{i, j}=\sigma\left(a_{i, j}\right)$ $(1 \leqslant i, j \leqslant m+1)$, an $(m+1) \times(m+1)$ system of matrix units in $\sigma(A)$ (unless $k=0$ in which case $F_{i, j}=0$ for all $i$ and $j$ ). Then

$$
T^{\sigma}(\cdot)=\sum_{i, j=1}^{m+1} F_{i, j}(\cdot) F_{i, j} \quad \text { and } \quad C^{\sigma}(\cdot)=\sum_{i, j=1}^{m+1} m F_{i, j}(\cdot) F_{j, i}-\sum_{i, j=1}^{m+1} F_{i, i}(\cdot) F_{j, j} .
$$

If $k=0$ then $T^{\sigma}=0=C^{\sigma}$. If $k>0$, then by Lemma 3.3 and the fact that compression by $\sum_{i=1}^{m+1} F_{i, i}$ is contractive and completely positive, we obtain that $\left\|T^{\sigma}\right\| \leqslant k \leqslant M_{\mathrm{U}}(\pi)$ and that $C^{\sigma}$ is $r$-positive whenever $r k \leqslant m$. Since $k \leqslant M_{\mathrm{U}}(\pi), C^{\sigma}$ is $r$-positive whenever $r M_{\mathrm{U}}(\pi) \leqslant m$.

Letting $\sigma$ vary in $\widehat{A / K}$ we conclude from $\left[\mathbf{9}\right.$, Lemma $1(\mathrm{v})$, (iv)] that $\left\|T_{A / K}\right\| \leqslant M_{\mathrm{U}}(\pi)$ and that $C_{A / K}$ is $r$-positive whenever $r \leqslant m / M_{\mathrm{U}}(\pi)$.

Let $A$ be a $\mathrm{C}^{*}$-algebra and $\pi \in \hat{A}$. Recall that

$$
S(\pi)=\{\sigma \in \hat{A}: \pi \text { and } \sigma \text { cannot be separated by disjoint open subsets of } \hat{A}\} \text {. }
$$

Theorem 3.6. Let $A$ be a $\mathrm{C}^{*}$-algebra and suppose that $\pi \in \hat{A}$ is such that $M_{\mathrm{U}}(\pi)=M<$ $\infty$ and $M<\operatorname{dim} \pi$. Suppose in addition that $\{\pi\}$ is relatively open in the set $S(\pi)$. Let $n$ be any positive integer such that $n<(\operatorname{dim} \pi) / M$.

(1) There exists an elementary operator $S$ on $A$ with coefficients in $A$ such that $\left\|S^{\pi}\right\|_{n M+1}>\|S\|_{n}$.

(2) There exists an $n$-positive elementary operator $R$ on $A$ with coefficients in $A$ such that $R^{\pi}$ is not $(n M+1)$-positive. 
Proof. By hypothesis, there is a closed two-sided ideal $I$ of $A$ such that $\pi \in \hat{I}$ and $\hat{I} \cap S(\pi)=\{\pi\}$. Thus $\pi$ is a separated point of $\hat{I}$. Let $m=n M$. Regarding $\pi$ as an element of $\hat{I}$, it is still the case that $M_{\mathrm{U}}(\pi)=M<\infty[8$, Lemma 2.7] and also $\operatorname{dim} \pi>m$. Let $J=I \cap \operatorname{ker} \pi$, a primitive and hence primal ideal of $I$. Applying Lemma 3.5 to $I, \pi$ and $J$, we obtain a $\tau_{s^{-}}$ neighbourhood $V$ of $J$ in $\operatorname{Id}(I)$, and elementary operators $T$ and $C$ on $I$, with coefficients in $I$, and a projection $E$ of rank $m+1$ in $\pi(I)$ such that

(i) $\left\|T^{\pi}\right\|_{m+1} \geqslant m+1$ and $C^{\pi}$ is not $(m+1)$-positive;

(ii) for each $K \in V,\left\|T_{I / K}\right\| \leqslant M_{\mathrm{U}}(\pi)$ and $C_{I / K}$ is $n$-positive;

(iii) $T^{\pi}(\cdot)=T^{\pi}(E(\cdot) E)$ and $C^{\pi}(\cdot)=C^{\pi}(E(\cdot) E)$.

Since $\pi$ is a separated point of $\hat{I}$, the identity map from $\operatorname{Prim}(I)$ with the Jacobson topology to $\left(\operatorname{Prim}(I), \tau_{s}\right)$ is continuous at $J[\mathbf{1 3}, 3.9 .4(\mathrm{a})]$. Thus $V \cap \operatorname{Prim}(I)$ contains a Jacobson-open neighbourhood $U$ of $J$ in $\operatorname{Prim}(I)$. There exists a closed two-sided ideal $L$ of $I$ such that $\operatorname{Prim}(L)=\{P \cap L: P \in U\}$ and, since $\pi \in \hat{L}$, there exists a positive element $c \in L$ such that $\|c\|=1$ and $\pi(c)=E$. We define elementary operators $S$ and $R$ on $A$ by

$$
S(x)=T(c x c)=\left(\left.T\right|_{L}\right)(c x c) \quad(x \in A)
$$

and

$$
R(x)=C(c x c)=\left(\left.C\right|_{L}\right)(c x c) \quad(x \in A) .
$$

It follows from (iii) that $\left.S^{\pi}\right|_{\pi(I)}=T^{\pi}$ and $\left.R^{\pi}\right|_{\pi(I)}=C^{\pi}$. Hence, by (i), $\left\|S^{\pi}\right\|_{m+1} \geqslant m+1$ and $R^{\pi}$ is not $(m+1)$-positive.

Let $\sigma$ be an irreducible representation of $L$ and let $\bar{\sigma}$ be the unique extension of $\sigma$ to an irreducible representation of $I$ on the Hilbert space $H_{\sigma}$. Then $K:=\operatorname{ker} \bar{\sigma} \in U \subseteq V$. Hence, since $\left(\left.T\right|_{L}\right)^{\sigma}=\left.\left(T^{\bar{\sigma}}\right)\right|_{\sigma(L)}$, we have $\left\|\left(\left.T\right|_{L}\right)^{\sigma}\right\| \leqslant\left\|T^{\bar{\sigma}}\right\| \leqslant\left\|T_{I / K}\right\| \leqslant M$. It follows from [9, Lemma $\left.1(\mathrm{v})\right]$ that $\left\|\left.T\right|_{L}\right\| \leqslant M$ and hence $\|S\| \leqslant M$. Hence, using [18, Exercise 3.10(ii)],

$$
\|S\|_{n} \leqslant n\|S\| \leqslant n M<m+1 \leqslant\left\|S^{\pi}\right\|_{m+1} .
$$

With $\sigma$ and $K$ as in the previous paragraph, we have $C_{I / K}$ is $n$-positive and hence so is $C^{\hat{\sigma}}$. Since $\left(\left.C\right|_{L}\right)^{\sigma}=\left.\left(C^{\bar{\sigma}}\right)\right|_{\sigma(L)}$, we obtain that $\left(\left.C\right|_{L}\right)^{\sigma}$ is $n$-positive. It follows from [9, Lemma 1(iv)] that $\left.C\right|_{L}$ is $n$-positive. Finally, $R=\left.\iota \circ C\right|_{L} \circ D$, where $D$ is the (completely positive) mapping from $A$ into $L$ given by $D(x)=c x c$ and $\iota: L \rightarrow A$ is the inclusion, and so $R$ is $n$-positive.

The proof of Theorem 3.6 shows that one may replace the condition on $S(\pi)$ by the assumption that $\pi$ is a separated point of $\hat{A}$, for then one may apply Lemma 3.5 directly to $A$ with $J=\operatorname{ker} \pi$. Alternatively, if $M_{\mathrm{U}}(\pi)<\infty$ (so that $\pi(A) \supseteq \mathcal{K}\left(H_{\pi}\right)$ ) and $\pi$ is a separated point of $\hat{A}$ then $\{\pi\}$ is automatically open in its closure $S(\pi)$. For a separable $\mathrm{C}^{*}$-algebra $A$, the set of separated points in $\hat{A}$ is a dense $G_{\delta}$ subset $[\mathbf{1 3}, 3.9 .4]$.

Corollary 3.7. Let $A$ be a $\mathrm{C}^{*}$-algebra and $n$ a positive integer. Suppose that $\pi \in \hat{A}$ and that $\operatorname{dim} \pi>n$.

(1) Suppose that $\left\|T^{\pi}\right\|_{n+1} \leqslant\|T\|_{n}$ for all elementary operators $T$ on $A$ with coefficients in A. Then $M_{\mathrm{U}}(\pi)>1$, that is, $\pi$ is not a Fell point.

(2) Suppose that for every n-positive elementary operator $T$ on $A$ with coefficients in $A, T^{\pi}$ is $(n+1)$-positive. Then $M_{\mathrm{U}}(\pi)>1$.

Proof. (1) Suppose that $M_{\mathrm{U}}(\pi)=1$. Then $\{\pi\}$ is relatively open in $S(\pi)$ [4, Lemma 4.3]. By Theorem 3.6(1) (with $M=1$ ), there exists an elementary operator $T$ on $A$ with coefficients in $A$ such that $\left\|T^{\pi}\right\|_{n+1}>\|T\|_{n}$. This contradicts the hypothesis.

The proof of (2) is similar, using Theorem 3.6(2). 
Combining Corollaries 3.2 and 3.7, we obtain the following result.

Theorem 3.8. Let $A$ be a $\mathrm{C}^{*}$-algebra, let $\pi \in \hat{A}$ and suppose that $\operatorname{dim} \pi>1$. The following conditions are equivalent:

(1) $M_{\mathrm{U}}(\pi)>1$ (that is, $\pi$ is not a Fell point);

(2) for all $n \geqslant 1$ and all elementary operators $T$ on $A,\left\|T^{\pi}\right\|_{n+1} \leqslant\|T\|_{n}$;

(3) there exists a positive integer $n<\operatorname{dim} \pi$ such that for all elementary operators $T$ on $A$ with coefficients in $A,\left\|T^{\pi}\right\|_{n+1} \leqslant\|T\|_{n}$;

(4) for all $n \geqslant 1$ and every $n$-positive elementary operator $T$ on $A, T^{\pi}$ is $(n+1)$-positive;

(5) there exists a positive integer $n<\operatorname{dim} \pi$ such that for every $n$-positive elementary operator $T$ on $A$ with coefficients in $A, T^{\pi}$ is $(n+1)$-positive.

\section{Factorial states and norms of elementary operators}

In this section, we shall apply Theorem 2.1 to give an alternative proof of [11, Theorem 4.5], and the results of Section 3 to give alternative proofs of [22, Corollary 4.5] and the corresponding parts of [21, Corollary 4.9]. We shall also need the following two propositions, which may be of independent interest.

Proposition 4.1. Let $A$ be a $\mathrm{C}^{*}$-algebra. The following conditions are equivalent:

(1) $M_{\mathrm{U}}(\pi)=\infty$ for all $\pi \in \hat{A}$;

(2) $M_{\mathrm{U}}(\pi)>1$ for all $\pi \in \hat{A}$;

(3) $A$ is antiliminal.

Proof. $\quad(2) \Rightarrow(3)$. Suppose that $A$ has a non-zero postliminal ideal $J$. Then $J$ contains a non-zero ideal $I$ with continuous trace $[\mathbf{1 9}, 6.2 .11]$. Let $\pi \in \hat{I}$. Then $\pi$ is a Fell point of $\hat{A}$ and so $M_{\mathrm{U}}(\pi)=1$ by [4, Corollary 4.2$]$.

$(3) \Rightarrow(1)$. Suppose that there exists $\pi \in \hat{A}$ such that $M_{\mathrm{U}}(\pi)<\infty$. Then by $[\mathbf{4}$, Proposition $2.3($ ii) $]$ there is a non-empty open subset $U$ of $\hat{A}$ such that $M_{\mathrm{U}}(\sigma)<\infty$ for all $\sigma \in \hat{A}$. Let $J$ be the non-zero ideal of $A$ such that $\hat{J}=U$. By [4, Remark 2.4(b); 8, Lemma 2.7], the upper multiplicity of each element of $\hat{J}$ (when computed relative to $\hat{J}$ ) is finite. Hence $J$ is liminal by $[4$, Corollary 4.9].

The next proposition is elementary but, perhaps, slightly surprising. It shows, in particular, that the notion of 'antiliminal-by-abelian' [9] is the same as 'abelian-by-antiliminal'.

Proposition 4.2. Let $A$ be a $\mathrm{C}^{*}$-algebra and $k$ a positive integer. The following conditions are equivalent:

(1) $A$ is $k$-subhomogeneous or $A$ is antiliminal or $A$ has a $k$-subhomogeneous ideal $I$ such that $A / I$ is antiliminal;

(2) $A$ is $k$-subhomogeneous or $A$ is antiliminal or $A$ has an antiliminal ideal $J$ such that $A / J$ is $k$-subhomogeneous;

Proof. $(1) \Rightarrow(2)$. Suppose that $A$ has a $k$-subhomogeneous ideal $I$ such that $A / I$ is antiliminal. Let

$$
U=\{\pi \in \hat{A}: \operatorname{dim} \pi>k\}
$$


a non-empty open subset of $\hat{A}[\mathbf{1 3}, 3.6 .3(\mathrm{i})]$. Let $J$ be the (non-zero) ideal of $A$ such that $\hat{J}=U$. Since $\hat{I}$ and $\hat{J}$ are disjoint, $I \cap J=\{0\}$. it follows that the quotient map $q_{I}: A \rightarrow A / I$ maps $J$ injectively onto an ideal of $A / I$. Hence $J$ is antiliminal. By construction,

$$
\widehat{A / J}=\{\pi \in \hat{A}: \operatorname{dim} \pi \leqslant k\}
$$

and so $A / J$ is $k$-subhomogeneous.

$(2) \Rightarrow(1)$. Suppose that $A$ has an antiliminal ideal $J$ such that $A / J$ is $k$-subhomogeneous. Let $V$ be the interior of $\widehat{A / J}$ (regarding the latter as a closed subset of $\hat{A}$ ) and let $I$ be the ideal of $A$ such that $\hat{I}=V$. Then $I$ is $k$-subhomogeneous (unless $V$ is empty in which case $I=\{0\})$ and $I \cap J=\{0\}$.

The quotient map $q_{I}: A \rightarrow A / I$ maps $J$ injectively onto an ideal of $A / I$. Moreover, $q_{I}(J)$ is an essential ideal of $A / I$ since $\hat{J}$ is dense in $\hat{A} / I$ (regarded as subsets of $\hat{A}$ ). Since $J$ is antiliminal so is $q_{I}(J)$ and hence so is $A / I$ (for, if $K$ were a non-zero liminal ideal of $A / I$, then $q_{I}(J) \cap K$ would be a non-zero liminal ideal of $\left.q_{I}(J)\right)$.

We now show how Theorem 2.1 and the results of Section 3 enable us to recapture the following combination of [11, Theorem 4.5; 21, Corollary 4.6; 22, Corollary 4.5].

Theorem 4.3. Let $A$ be a $\mathrm{C}^{*}$-algebra and $n$ a positive integer. The following conditions are equivalent:

(1) $F_{n}(A)$ is weak ${ }^{*}$-dense in $F_{\infty}(A)$;

(2) $F_{n}(A)$ is weak*-dense in $F_{n+1}(A)$;

(3) $A$ is $n$-subhomogeneous or $A$ is antiliminal or $A$ has a $n$-subhomogeneous ideal $I$ such that $A / I$ is antiliminal;

(4) $\|T\|_{c b}=\|T\|_{n}$ for every elementary operator $T$ on $A$;

(5) $\|T\|_{n+1}=\|T\|_{n}$ for every elementary operator $T$ on $A$ with coefficients in $A$;

(6) every $n$-positive elementary operator on $A$ is completely positive;

(7) every n-positive elementary operator on $A$ with coefficients in $A$ is $(n+1)$-positive.

Proof. $\quad(1) \Rightarrow(2)$. This is immediate.

(2) $\Rightarrow(3)$. Let $U=\{\pi \in \hat{A}: \operatorname{dim} \pi>n\}$, an open subset of $\hat{A}$. If $U$ is empty then $A$ is $n$-subhomogeneous.

Suppose that $U$ is non-empty and let $J$ be the ideal of $A$ such that $\hat{J}=U$. Let $\pi \in U$. Since $\operatorname{dim} \pi>n$, there exists $\phi \in F_{n+1}(A) \backslash F_{n}(A)$ such that $\theta(\phi)=\pi$. By $(2), \phi \in \overline{F_{n}(A)}$. It follows from Theorem $2.1((3) \Rightarrow(4))$ that $M_{\mathrm{U}}(\pi)>n / n=1$. By [4, Remark 2.4(b); 8, Lemma 2.7], the upper multiplicity of each element of $\hat{J}$ (when computed relative to $\hat{J}$ ) is greater than 1 . By Proposition 4.1, $J$ is antiliminal. If $J \neq A$ then, by definition of $U, A / J$ is $n$-subhomogeneous.

We have shown that $A$ satisfies condition (2) of Proposition 4.2 and so, by that proposition, condition (3) holds.

$(3) \Rightarrow(1)$. Since $F_{f}(A)$ (the set of type-I factorial states of finite degree) is norm-dense in $F_{\infty}(A)$, it suffices to show that $F_{f}(A) \subseteq \overline{F_{n}(A)}$. If $A$ is $n$-subhomogeneous then $F_{f}(A)=$ $F_{n}(A)$. Therefore, we may assume that $A / I$ is antiliminal where either $I=\{0\}$ or $I$ is an $n$-subhomogeneous ideal of $A$.

Suppose that $\phi \in F_{f}(A) \backslash F_{n}(A)$ and let $\theta(\phi)=\pi$. Since $\phi \notin F_{n}(A), \pi \in \widehat{A / I}$. By Proposition $4.1, M_{\mathrm{U}}(\pi)=\infty$ (in $\widehat{A / I}$ and hence in $\hat{A}$ ). There exists $m \geqslant n$ such that $\phi \in F_{m+1}(A) \backslash$ $F_{m}(A)$. Hence $\operatorname{dim} \pi>m$. Since $M_{\mathrm{U}}(\pi)=\infty>m / n$, it follows from Theorem $2.1((4) \Rightarrow(2))$ that $\phi \in \overline{F_{n}(A)}$.

$(3) \Rightarrow(4)$. Let $T$ be an elementary operator on $A$. By (3) and [9, Lemma 2(ii)], we may reduce to the two cases where $A$ is either $n$-subhomogeneous or antiliminal. Suppose first that 
$A$ is $n$-subhomogeneous. For $\pi \in \hat{A}$, we have $\left\|T^{\pi}\right\|_{c b}=\left\|T^{\pi}\right\|_{n}$ [18, Proposition 8.10]. Then (4) follows by taking the supremum over $\pi$ and using [9, Lemma 1(v)].

Suppose now that $A$ is antiliminal and $m \geqslant n$. Let $\pi \in \hat{A}$. By Proposition $4.1, M_{\mathrm{U}}(\pi)=\infty$. Hence, by Theorem 3.1(1), $\left\|T^{\pi}\right\|_{m} \leqslant\|T\|_{n}$. Taking the supremum over $\pi \in \hat{A}$, we obtain that $\|T\|_{m} \leqslant\|T\|_{n}[\mathbf{9}$, Lemma $1(\mathrm{v})]$. Finally, taking the supremum over $m \geqslant n$, we obtain that $\|T\|_{c b}=\|T\|_{n}$.

$(4) \Rightarrow(5)$. This is immediate.

$(5) \Rightarrow(2)$. If $F_{n+1}(A)=F_{n}(A)$, then there is nothing to prove, so suppose that $\phi \in F_{n+1}(A) \backslash$ $F_{n}(A)$ and let $\pi=\theta(\phi)$. Then $\operatorname{dim} \pi>n$. Assuming (5), and using [9, Lemma 1(v)] again, we have

$$
\left\|T^{\pi}\right\|_{n+1} \leqslant\|T\|_{n+1}=\|T\|_{n}
$$

for all elementary operators $T$ on $A$ with coefficients in $A$. By Corollary 3.7(1), $M_{\mathrm{U}}(\pi)>1$. By Theorem $2.1((4) \Rightarrow(2)), \phi \in \overline{F_{n}(A)}$.

$(3) \Rightarrow(6)$. Let $T$ be an $n$-positive elementary operator on $A$. By (3) and [9, Lemma 2(i)], to show that $T$ is completely positive we may reduce to the two cases where $A$ is either $n$-subhomogeneous or antiliminal. Suppose first that $A$ is $n$-subhomogeneous. For $\pi \in \hat{A}$, we have $T^{\pi}$ is $n$-positive by $[\mathbf{9}$, Lemma $1(\mathrm{iv})]$ and $\pi(A)$ is ${ }^{*}$-isomorphic to $M_{r}(\mathbb{C})$ for some $r \leqslant n$. Hence $T^{\pi}$ is completely positive by [18, Theorem 6.1]. Hence $T$ is completely positive by $[\mathbf{9}$, Lemma 1(iv)].

Suppose now that $A$ is antiliminal and $m \geqslant n$. Let $\pi \in \hat{A}$. By Proposition $4.1, M_{\mathrm{U}}(\pi)=\infty$. Hence, by Theorem 3.1(2), $T^{\pi}$ is $m$-positive. Since this holds for all $\pi \in \hat{A}$, it follows from [9, Lemma 1 (iv)] that $T$ is $m$-positive as required.

$(6) \Rightarrow(7)$. This is immediate.

$(7) \Rightarrow(2)$. This is similar to the proof that (5) implies (2), but uses $[\mathbf{9}$, Lemma 1(iv)] and Corollary $3.7(2)$.

\section{References}

1. P. Ara and M. Mathieu, Local multipliers of $\mathrm{C}^{*}$-algebras (Springer, London, 2003).

2. R. J. Archbold, 'On factorial states of operator algebras', J. Funct. Anal. 55 (1984) 25-38.

3. R. J. Archbold, 'Limits of pure states', Proc. Edinburgh Math. Soc. 69 (1989) 249-254.

4. R. J. Archbold, 'Upper and lower multiplicity for irreducible representations of $\mathrm{C}^{*}$-algebras', Proc. London Math. Soc. 69 (1994) 121-143.

5. R. J. Archbold and C. J. K. BAtty, 'On factorial states of operator algebras III', J. Operator Theory 15 (1986) 53-81.

6. R. J. Archbold and K. Deicke, 'Bounded trace C*-algebras and integrable actions', Math. Z. 250 (2005) 393-410

7. R. J. Archbold and J. S. Spielberg, 'Upper and lower multiplicity for irreducible representations of C*-algebras II', J. Operator Theory 36 (1996) 201-231.

8. R. J. Archbold, D. W. B. Somerset and J. S. Spielberg, 'Upper multiplicity and bounded trace ideals in C*-algebras', J. Funct. Anal. 146 (1997) 430-463.

9. R. J. Archbold, M. Mathieu and D. W. B. Somerset, 'Elementary operators on antiliminal C*-algebras', Math. Ann. 313 (1999) 609-616.

10. R. J. Archbold, D. W. B. Somerset and R. M. Timoney, 'Completely bounded mappings and simplicial complex structure in the primitive ideal space of a $\mathrm{C}^{*}$-algebra', Trans. Amer. Math. Soc., to appear.

11. C. J. K. BAtтY and R. J. ARchbold, 'On factorial states of operator algebras II', J. Operator Theory 13 (1985) 131-142.

12. M. D. Chor, 'Positive linear maps on C*-algebras', Canadian J. Math. 24 (1972) 520-529.

13. J. Dixmier, Let $\mathrm{C}^{*}$-algèbres et leurs représentations (Gauthier-Villars, Paris, 1964).

14. J. M. G. FeLL, 'The structure of algebras of operator fields', Acta Math. 106 (1961) 233-280.

15. J. GLimm, 'A Stone-Weierstrass theorem for C*-algebras', Ann. Math. 72 (1960) 216-244.

16. J. Glimm, 'Type I C*-algebras', Ann. Math. 73 (1961) 572-612.

17. A. J. LAzAR, 'C $C^{*}$-bundles for certain liminal $C^{*}$-algebras', Integr. Equ. Oper. Theory 60 (2008) 381-404.

18. V. PAulsen, Completely bounded maps and operator algebras (Cambridge University Press, Cambridge, 2002).

19. G. K. Pedersen, ' $C^{*}$-algebras and their automorphism groups (Academic Press, London, 1979). 
20. F. W. Shultz, 'Pure states as a dual object for $C^{*}$-algebras', Comm. Math. Phys. 82 (1982) 497-509.

21. R. M. Timoney, 'An internal characterization of complete positivity for elementary operators' Proc. Edinburgh Math. Soc. 45 (2002) 285-300.

22. R. M. Timoney, 'Computing the norms of elementary operators', Illinois Math. 47 (2003) 1207-1226.

23. R. M. Timoney, 'Some formulae for norms of elementary operators', J. Operator Theory 57 (2007) 121-145.

24. J. Tomiyama and M. TAKesAKI, 'Applications of fibre bundles to the certain class of $C^{*}$-algebras', Tohoku Math. J. (2) 13 (1961) 498-523.

Robert J. Archbold and Douglas W. B. Somerset

Department of Mathematical Sciences University of Aberdeen

Aberdeen

AB24 3UE

United Kingdom

r.archbold@maths.abdn.ac.uk

somerset@quidinish.fsnet.co.uk
Richard M. Timoney

School of Mathematics

Trinity College

Dublin 2

Ireland

richardt@maths.tcd.ie 